\title{
Effects of cannabinoid agonists on the proteome of a human oligodendrocyte culture: possible implications for schizophrenia
}

\author{
Priscilla R. Rumin*, Valéria de Almeida, Daniel Martins-de-Souza.
}

\begin{abstract}
Several studies have suggested the involvement of the endocannabinoid system in the pathophysiology of schizophrenia. In addition, it also showed that cannabinoids can affect the function of oligodendrocytes. In order to better understand the molecular mechanisms of involved, we had investigated the effects of endocannabinoids and synthetic cannabinoid agonists on a human oligodendrocyte cell line (MO3.13). Using proteomic tools and in silico analyses, we found alterations in cannabinoid-treated cells previously associated to the disease. Also, we found alterations in biological processes, such as cell cycle, mRNA processing and metabolism, and cellular component organization. These are potentially interesting to be explored in a alternative cannabinoid-centered therapeutic strategy. Taken together, our results may contribute to the elucidation of the role of endocannabinoid system in oligodendrocytes and the possible implications for undertanding the pathophysiology of schizophrenia.
\end{abstract}

Key words: Endocannabinoid System, Proteomics, Glia

\section{Introduction}

Schizophrenia is a severe disorder caused from an interaction between neurodevelopmental processes and environmental factors. ${ }^{1}$ As a multifactorial disease, a number of neuronal abnormalities implicate in the symptoms, including defects in neurotransmission. More recently, studies have suggested the possible involvement of the endocannabinoid system. ${ }^{2}$

Cannabinoids also seems to play a role in the regulation of glial cells, important components in the pathophysiology of the disease, especially related to neuronal differentiation and survival ${ }^{3}$. Alterations as reduction of the density of oligodendrocytes and white matter in schizophrenia patients, that may occur during the proliferation and maturation of these progenitors cells, may damage neural function related to defects in neurotransmission found on the desease. ${ }^{4}$

This work aims to analyze the effect of endocannabinoids and synthetic cannabinoids in the proteome of human oligodendrocytes in culture; in order to identify proteins differentially expressed by these different treatments, their interaction networks and altered biochemical pathways, besides possible reversions of effects in a model of glutamatergic hypofunction. Aiming to delineate the role of endocannabinoid system at a better understanding of schizophrenia.

\section{Results and Discussion}

Cultured oligodendrocytes (MO3.13) were treated with the endocannabinoids Anandamide (AEA) and 2-arachidonylglycerol (2-AG) and with synthetic cannabinoids WIN55,212-2, ACEA and HU308. These drugs also used in a co-treatment with MK-801, an NMDA antagonist, which potentially induce schizophrenia-like symptoms. Treated cells were submitted to liquid chromatography coupled to mass spectrometry. Identified proteins were quantified by the Progenesis QI, and further analyzed in silico using String (version 10.5 and 11.0) and Reactome (version 3.6).

Treatments with all synthetic cannabinoids induced similar differences in biological processes as cell cycle, mRNA processing and metabolism. These are interesting considering the effects of cannabinoids in cell survival, proliferation and differentiation observed other in vivo and in vitro studies. Synthetic cannabinoids also induced the differential expression of IQEC2, YWHAE and SDF4, which were previously associated to psychiatric disorders. 5,6 .

Endocannabinoids-treated cells modulated the expression of 75 differentially regulated proteins. Eight of those were downregulated and have been previously associated to schizophrenia (ATP6V1A, ATP5B, APOA4, A2SM, AHSG, ALB, YWHAG and SEPT11). ${ }^{7}$ Altered proteins play roles in myelin sheath, actin cytoskeleton, eukaryotic translation initiation factor 3 and spliceosomal complex. In addition,proteins specifically modulated by the different treatments also bring potentially interesting therapeutic targets, which must be more explored.

\section{Conclusions}

Results are still initial, but show potential targets to be explored. In this way, this study tried to aggregate date to a deeper understanding of the role of the endocannabinoid system in oligodendrocyte function in the context of schizophrenia.

\section{Acknowledgement}

To the SAE/UNICAMP, by the scholarship granted, as well as the support for the laboratory during this study by FAPESP, CNPQ, and Instituto Serrapilheira.

\footnotetext{
${ }^{1}$ Owen M.J., et al. 'Schizophrenia”.Lancet. 2016, 388(10039), 86-97.

${ }^{2}$ Fernandez-Espejo E., et al. "Role of cannabis and endocannabinoids in the genesis of schizophrenia." Psychopharmacology. 2009. 206(4), 531-549.

${ }^{3}$ de Almeida V., Martins-de-Souza D. "Cannabinoids and glial cells: possible mechanism to understand schizophrenia." European archives of psychiatry and clinical neuroscience.2018, 1-11

${ }^{4}$ Cassoli J.S., et al. "Disturbed macro-connectivity in schizophrenia linked to oligodendrocyte dysfunction: from structural findings to molecules." npj Schizophrenia. 2015, 1, 15034.

${ }^{5}$ Saia-Cereda, Verônica M., et al. "The Nuclear Proteome of White and Gray Matter from Schizophrenia Postmortem Brains." Molecular neuropsychiatry. 2017, 3.1, 37-52.

Wang, Yuan, et al. "Blood-based dynamic genomic signature for obsessivecompulsive disorder." Am. Journal of Medical Genetics.2018, 177.8, 709-716.

${ }^{8}$ Davalieva, Katarina, et al. "Proteomics research in schizophrenia." Frontiers in cellular neuroscience.2016, 10, 18.
} 